
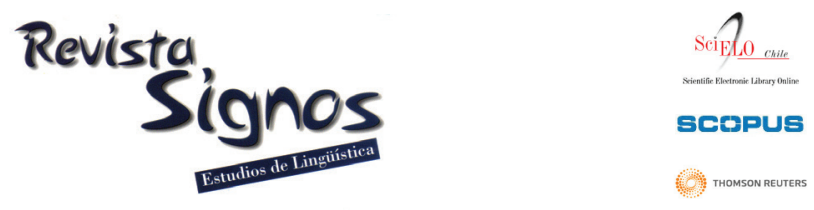

\title{
Bueno, pues, es que... en fin: ¿Qué marcadores discursivos enseñar?
}

\author{
Bueno, pues, es que... en fin: What discourse markers \\ should we teach?
}

Noemí Domínguez García

UniVERSidAd DE SALAMANCA

ESPAÑA

noedom@usal.es

Recibido: 04-II-2014 / Aceptado: 19-VI-2015

\section{Resumen}

El objetivo de este trabajo es identificar los marcadores habituales que organizan tanto la conversación coloquial como los discursos formales, ya sean orales o escritos. Para ello, nos basaremos en un análisis de dos corpus de conversaciones y de un corpus de discursos escritos breves. Estos corpus se reducen a la variedad geográfica del español de España y, por tanto, las conclusiones a las que lleguemos son solo el comienzo de una propuesta de investigación futura más amplia. Tras hacer un análisis de frecuencia de aparición de los marcadores localizados en estos tres corpus, revisamos las listas y clasificaciones de marcadores en las gramáticas y diccionarios considerados de referencia para comprobar el escaso o nulo uso de muchos de ellos como sucede, por ejemplo, con los digresores o los comentadores. También el análisis de frecuencia realizado, cruzado con la variable de tipo de discurso, oral/escrito, y de registro, coloquial/formal, arroja algunas conclusiones predecibles, como la preferencia de los estructuradores de la información por los discursos escritos y de los conversacionales por los discursos orales; pero también muestra conclusiones novedosas, como la frecuencia de aparición de los ordenadores de cierre en las conversaciones coloquiales, o de los conversacionales evidenciales en los discursos escritos. Finalmente, es nuestra intención aplicar estudios como el desarrollado en este artículo a la elaboración de listas de marcadores que corroboren y amplíen las diseñadas en los planes curriculares de aprendizaje de español como segunda lengua o como lengua extranjera.

Palabras Clave: Marcadores del discurso, español como lengua extranjera, tipologías textuales, registros discursivos. 


\begin{abstract}
This paper focuses on the identification of the usual discourse markers that organise both formal and informal interactions, either in oral or written speech. Drawing on two corpora for oral conversations and one corpus for written speech, the material is only based on the geographical variation of the Spanish language as produced in Spain. Therefore, the conclusions to be reached would be only the beginning of a wider research project to be developed further in the near future. After a data-frequency analysis, findings were compared with the ways in which markers are most commonly listed and classified in reference dictionaries and grammar books to confirm the little or no use of many of those markers, such as the digressive or the 'here-and-now' markers. In addition, crossing the data-frequency collected with the variables type of speech -oral or written- and register -formal or colloquial- some predictable conclusions could be drawn, i.e., the preference of written speech for discourse organisers or the conversation markers tendency to oral interactions. The analysis also allowed for new conclusions, such as the use of closing markers in colloquial speech or the conversation-evidential markers in written discourses. Finally, we would like to apply analyses like the one collected in this study to obtain a new list of markers to support and extend those already taught for the acquisition of discourse competence in Spanish as a Foreign Language.
\end{abstract}

Key Words: Discourse markers, Spanish as a Foreign Language, textual tipologies, discourse register.

\title{
INTRODUCCIÓN
}

Este trabajo propone como objetivo la identificación de los marcadores habituales que organizan la conversación coloquial y los discursos formales, ya sean orales o escritos. Comparemos, a tal efecto, los ejemplos (1), (2) y (3) siguientes y prestemos atención a las palabras marcadas en cursiva:

(1) $\mathrm{E}: h h$

G: pues bueno puess [a lo que iba=]

L: [pues jvaya tela!]

G: = el chaval este/ o sea $\rightarrow /$ pues/ una día $\uparrow$ un día $\uparrow$ ¿no?/ decidió apuntarse a la autoescuela $\uparrow / y$ se apuntó a la autoescuela/ o sea bueno creo que se salió enen sexto/ no ha terminao nii-ni la Egebé ¿no? se fue con su padre a trabajar $\$$ (L.15.A.2, en Briz \& Grupo Val.Es.Co., 2002:112) ${ }^{1}$

El menor grado de planificación del discurso en (1) se plasma, además de en la repetición de secuencias ('pues bueno puess', 'nii-ni'), en el uso de determinadas clases de marcadores, como los metadiscursivos con funciones demarcativas ("pue(s) 
s', 'o sea'2), o los regresivos ('a lo que iba'3); la proximidad de los participantes y la finalidad interpersonal de la interacción quedan reflejadas en el uso de marcadores polifuncionales en usos metadiscursivos o modales ('bueno', 'hh'), o de enfocadores de la alteridad y apéndices comprobativos (“¿no?’); además, se dan combinaciones de todos ellos ('pues bueno puess a lo que iba', 'o sea pues', 'o sea bueno'). Si añadimos la cotidianidad del tema tratado -el hecho de que un chico que había fracasado en los estudios aprobara a la primera el carné de conducir- y el tono informal - ¡vaya tela!' formas sincopadas de los participios, énfasis prosódico en algunas palabras-, la conclusión a la que llegamos es que nos hallamos ante un caso típico de discurso oral conversacional coloquial.

(2) Su periódico, por el titular de ese día (La Iglesia puso trabas a la paz, y el Ejército, no, basado en las declaraciones del presidente), parece dudar de dos cosas. En primer lugar, que esas dudas razonables en las organizaciones de derechos humanos y en la Iglesia no son para ustedes ni importantes ni dignas de tener en cuenta (¿por qué?). En segundo lugar, dudan de que los lectores seamos dignos de ser respetados con una información objetiva, ya que son la Iglesia y las organizaciones de derechos humanos las que denuncian una paz sin justicia, no solo la Iglesia. (El País, 7/2/1997, en Domínguez, 2002: 609).

Por el contrario, la mayor planificación de la información contenida en el discurso de (2) queda clara en el uso de ordenadores discursivos dispuestos en una serie correlativa ('en primer lugar, en segundo lugar'). Esta planificación se anuncia, además, desde el primer enunciado, con otras unidades no marcadoras ('dos cosas') que lo vinculan con todo lo que sigue. Si a ello añadimos la ausencia física de los interlocutores y la finalidad transaccional del texto, concluiremos que nos hallamos ante un ejemplo típico de discurso escrito.

(3) ENC. Aparte de la cuestión académica, ¿cuál era el ambiente estudiantil? ¿Es diferente -tú crees-al que existe ahora en la universidad?

INF. Pues era totalmente distinto, C. Era totalmente distinto. Psss..., del año sesenta y cuatro al sesenta y nueve, o sea, es franquismo pleno por un lado, pero, por otra parte, es una España que, en principio, es mucho más rural, y una universidad muchísimo más elitista ( $n^{\circ}$ 9, en Fernández Juncal, 2005: 177)

Tratándose, como en el ejemplo (1), de un discurso oral, el ejemplo (3) difiere de aquel en que es una entrevista y, como tal, se caracteriza por la asimetría, tanto del papel de los participantes como de la longitud de sus turnos, así como por el uso de la estructura pregunta-respuesta que adopta ('Tusón, 1997). Lo incluimos en el grado '+oral + formal'. Y si nos fijamos en su organización cohesiva, aunque observamos marcas típicas del discurso oral como el marcador 'pues' para iniciar respuesta, o rellenadores interjectivos del tipo 'psss', se acerca a la planificación del discurso escrito del ejemplo (2), por cuanto introduce marcadores discursivos que organizan 
sus argumentos ('por un lado', 'pero por otra parte'), un reformulador explicativo ('o sea') o un operador de debilitamiento argumentativo ('en principio's).

Con estos ejemplos queremos poner de manifiesto, además de las diferencias entre tipos de discurso y tipos de registro, el que será objetivo de este trabajo: la identificación de los marcadores más frecuentes que organizan la conversación coloquial y de los más usados en el registro formal, ya sea oral o escrito. Nuestra intención con este análisis es la de obtener una nueva lista de marcadores que se organice por criterios de frecuencia de uso en los distintos tipos de discurso y de registro para su aplicación a la enseñanza de español como lengua extranjera. Con ello contribuiremos a resolver el problema de aplicación a la enseñanza de español como lengua extranjera que presentan las listas habituales (Martín Zorraquino \& Portolés, 1999; Briz, Pons \& Portolés 2008; Fuentes, 2009), demasiado exhaustivas porque incluyen todos los marcadores que utilizaría un hablante nativo de español, independientemente del medio y registro discursivos ${ }^{5}$. Con la lista de marcadores obtenida tras nuestro análisis podríamos distribuir el aprendizaje de los marcadores discursivos más frecuentes -al menos en el español de España- en los distintos niveles de referencia - A1-C2-.

A este respecto, Holgado (2014) señala la escasa atención que hasta ahora se ha dedicado a los marcadores del discurso en el ámbito de la enseñanza de español como lengua extranjera y recopila la bibliografía existente sobre el tema. Es cierto que en trabajos recientes (Albelda, 2005) ya se aprecian intentos de aplicación de los estudios de marcadores del discurso en español como lengua materna a la enseñanza de español como lengua extranjera, pero aún queda un largo camino por recorrer. Y en ese camino este trabajo pretende ser una aportación.

En cuanto al marco teórico en el que este estudio se inserta -el estudio de los marcadores del discurso en el español como lengua materna-, así como la metodología que emplearemos para obtener la lista de marcadores más frecuentes en el discurso escrito y oral y en los registros formal y coloquial -la obtención de datos reales a través de tres corpus de referencia-, los apartados que siguen darán cuenta de ello.

\section{Marco teórico}

Desde el aumento, en las últimas décadas del siglo pasado, del interés por el estudio del discurso, han sido muchos los autores esforzados en señalar la importancia del medio, fónico o gráfico, a través del cual se realizan nuestras manifestaciones lingüísticas, como rasgo diferenciador de los tipos de discurso ${ }^{6}$. Al respecto, Marimón Llorca (2008), reproduciendo el esquema de Eggins (2002 apud Marimón Llorca, 2008) sobre algunas situaciones comunicativas a las que aplica las variables de contacto visual de los interlocutores, canal de comunicación empleado e inmediatez en la respuesta, obtiene la siguiente gradación como se muestra en la Tabla 1: 
Tabla 1. Diferencias entre discursos orales y escritos (Marimón Llorca, 2008).

\begin{tabular}{|c|c|c|c|c|c|c|}
\hline Variables & $\begin{array}{c}\text { Conversación } \\
\text { coloquial }\end{array}$ & Teléfono & $\begin{array}{c}\text { Correo } \\
\text { electrónico }\end{array}$ & Fax & $\begin{array}{c}\text { [comunicación } \\
\text { por] Radio }\end{array}$ & Novela \\
\hline Contacto visual & + & - & - & - & - & - \\
\hline Canal auditivo & + & + & - & - & + & - \\
\hline Respuesta inmediata & + & + & \pm & \pm & \pm & - \\
\hline
\end{tabular}

A esta gradación debemos añadir la que se da cuando incluimos la variable del registro, para la que admitimos sin reservas la existencia de un continum que va desde lo '+coloquial' a lo '+formal' ('Tusón, 1997; Briz, 1998).

En cuanto a los marcadores del discurso, Pons (1998) ya recogía setenta definiciones de 'marcador del discurso' a las que clasificó en cuatro grupos según incidieran en la unión de enunciados, en la unión de argumentos, en el hecho de implicar algo más que la unión o en señalar su ámbito de operación en la estructura de la conversación. Un año más tarde, en la Gramática descriptiva de la lengua española dirigida por Bosque y Demonte, se incluyó un capítulo dedicado a los marcadores del discurso (Martín Zorraquino \& Portolés, 1999). La lista de marcadores que incluye será la que tomaremos como referencia en este artículo.

En dicho capítulo de la Gramática descriptiva, los marcadores del discurso se definían como:

"unidades lingüísticas invariables, [que] no ejercen una función sintáctica en el marco de la predicación oracional -son, pues, elementos marginales-y [que] poseen un cometido coincidente en el discurso: el de guiar, de acuerdo con sus distintas propiedades morfosintácticas, semánticas y pragmáticas, las inferencias que se realizan en la comunicación” (Martín Zorraquino \& Portolés, 1999: 63).

Idéntica definición había aparecido en la obra de Portolés (1998) y suponía la culminación de décadas de investigaciones que conocieron en la tesis de Blakemore (1987) un punto de inflexión (Portolés, 2004). A partir de las obras de Fuentes (1987) y de Mederos (1988) se sucede en el ámbito de la lingüística española un sinnúmero de artículos y monografías que se dedican al análisis detallado de un determinado marcador o a la caracterización de un grupo específico de marcadores?.

Algunos estudios añaden al análisis y clasificación de los marcadores del discurso notas sobre su registro de aparición más frecuente. En ese sentido, es merecidamente destacable el esfuerzo del Diccionario de Particulas Discursivas del Español (en línea, Briz et al., 2008) -en adelante, DPDE- por marcar el uso en registros formales o coloquiales de las partículas que incluye en su obra. Esfuerzo análogo destacamos en el Diccionario de conectores y operadores del español (Fuentes, 2009). En este artículo tendremos en cuenta las conclusiones sobre el registro a las que llegan estas obras e indicaremos aquellos casos en los que un determinado marcador registrado como 'formal' en 
las obras citadas aparece en el corpus de conversaciones coloquiales que tomamos como referencia, así como los casos de aparición de marcadores registrados como 'coloquiales' en los corpus escrito y conversacional formal de referencia. Señalaremos si se trata de un uso esporádico, idiosincrásico de un determinado informante, o si viene determinado por el tipo de secuencia textual en el que se inserta.

Finalmente, no olvidamos nuestra pretensión de aplicar esta propuesta a la enseñanza de español como lengua extranjera. A este propósito, tomaremos como base la distribución del aprendizaje de los marcadores del discurso por niveles que determina el Plan Curricular del Instituto Cervantes (2006 y en línea) -en adelante, PCIC-, de manera que, a modo de ejemplo, podremos comparar si los marcadores conversacionales coloquiales de control de contacto que incluye el PCIC en los niveles A1 y A2 ('¿no?', '¿eh?', 'oiga', 'oye', 'mira', 'mire') coinciden con los marcadores más frecuentes de control de contacto en nuestro estudio, o si los que incluye en el nivel B1 ('‘sabes?’, ‘'ves?’, ‘¿entiendes?’) son menos frecuentes que los anteriores ${ }^{8}$.

\section{Marco metodológico}

Como señalábamos en la Introducción, hemos seleccionado tres corpus de referencia para nuestro análisis: el 'Corpus de conversaciones coloquiales' creado por Briz y el Grupo Val.Es.Co. (2002) -en adelante, Val.Es.Co-; el 'Corpus de Habla Culta de Salamanca' elaborado por Fernández Juncal (2005) -en adelante CHCS-; y el corpus de Cartas al Director del diario El País, en Domínguez García (2002) -al que aludiremos como 'corpus escrito'-. Los corpus orales recogen diecinueve conversaciones y catorce entrevistas, respectivamente, en tanto que el corpus escrito cuenta con cuatrocientas cartas de no más de treinta líneas mecanografiadas?.

Las conversaciones coloquiales de Val.Es.Co duran una media de 22 minutos y 4.790 palabras, siendo las más breves de 7 minutos y las más extensas de $50^{10}$. Las entrevistas del CHCS contienen una media de 5.361 palabras, siendo la más breve de 4.660 y la más extensa de 6.285. Las entrevistas son géneros discursivos semiformales que pueden contener secuencias coloquiales según el tema que se esté tratando en ellas, o el tono que adopte la entrevista en su decurso. En este sentido, somos conscientes del riesgo que entraña la localización de marcadores coloquiales en el CHCS, que hemos tomado como referencia de registro oral formal, ya que puede llevarnos a conclusiones erróneas sobre la presencia de elementos coloquiales en el registro formal. Nos esforzaremos en este artículo en minimizar dichos riesgos prestando especial atención al contexto secuencial de aparición de los marcadores del CHCS.

Seleccionados los corpus de referencia, el segundo paso ha sido el rastreo en ellos de los marcadores conversacionales y de los estructuradores de la información, siguiendo la terminología mayoritariamente aceptada ${ }^{11}$. Para los marcadores conversacionales, los comentadores y los digresores -subtipos de estructuradores de la información-, 
seguimos la lista de Martín Zorraquino y Portolés (1999)²; para los organizadores seguimos una lista más reciente, de Garcés (2008).

En los apartados que siguen, presentaremos tablas con el número de apariciones de cada marcador en cada uno de los corpus de referencia. Analizaremos sus frecuencias absolutas y sus frecuencias relativas tomando como cálculo de base el número de apariciones de cada marcador en relación a los marcadores de su corpus y en relación a su aparición en todos los corpus. Puede darse el caso de que un marcador sea especialmente frecuente en un informante o en un autor de las cartas del corpus de discursos escritos, lo que provocaría un dato aberrante a la hora de analizar las frecuencias. Sin embargo, este hecho no ha sido significativo, por lo que los resultados del análisis son, para estos tres corpus y en cierto modo, concluyentes. Una ampliación de la muestra que se toma como referencia podrá aportar conclusiones más determinantes sobre el uso de los marcadores del discurso en la variedad geográfica del español de España. Sin embargo, reservaremos esta ampliación para un posterior estudio. El análisis incluido en este artículo, así como sus conclusiones, deben tomarse solo como una primera aproximación al estudio de los marcadores más usados por los hispanohablantes españoles.

\section{Resultados y discusión}

\subsection{Marcadores conversacionales}

Casi puede asegurarse que el lugar de aparición mayoritaria de estos marcadores es la conversación coloquial y, por tanto, dominarán en el corpus de Val.Es.Co. Así es, pero las preguntas que nos hacemos son: ¿cuáles de los marcadores habitualmente incluidos en la lista de marcadores conversacionales son los más frecuentes?; ¿cuáles de estos marcadores aparecen también en el discurso oral formal que representa el CHCS?; finalmente, ¿hay algún indicio de coloquialidad en el corpus escrito que hemos utilizado?

Los resultados obtenidos se presentan en la Tabla 2: 
Tabla 2. Frecuencias absolutas y relativas (\%) de los marcadores conversacionales en su propio corpus/ respecto a los tres corpus.

\begin{tabular}{|c|c|c|c|c|c|c|}
\hline Marcador & $\begin{array}{l}\text { Oral, } \\
\text { coloquial }\end{array}$ & $\%$ & $\begin{array}{l}\text { Oral, } \\
\text { formal }\end{array}$ & $\%$ & $\begin{array}{l}\text { Escrito, } \\
\text { formal }\end{array}$ & $\%$ \\
\hline $\mathrm{Al}$ parecer & 0 & 0 & 0 & 0 & 8 & $6,67 / 100$ \\
\hline Bien & 47 & $2,11 / 94$ & 2 & $0,11 / 4$ & 1 & $0,83 / 2$ \\
\hline Bueno $^{13}$ & 253 & $11,38 / 45,59$ & 300 & $16,11 / 54,05$ & 2 & $1,67 / 0,36$ \\
\hline Claro & 266 & $11,96 / 40,29$ & 137 & $7,36 / 33,25$ & 9 & $7,5 / 2,18$ \\
\hline De acuerdo & 0 & 0 & 1 & $0,05 / 100$ & 0 & 0 \\
\hline Desde luego & 10 & $0,45 / 28,57$ & 13 & $0,70 / 37,14$ & 12 & $10 / 34,28$ \\
\hline Efectivamente & 2 & $0,09 / 25$ & 1 & $0,05 / 12,5$ & 5 & $4,17 / 62,5$ \\
\hline ¿Eh? & 237 & $10,66 / 72,70$ & 88 & $4,73 / 26,99$ & 1 & $0,83 / 0,31$ \\
\hline En efecto & 0 & 0 & 0 & 0 & 4 & $3,33 / 100$ \\
\hline ¿Entiendes? & 6 & $0,18 / 60$ & 4 & $0,21 / 40$ & 0 & 0 \\
\hline Escucha & 4 & $0,18 / 100$ & 0 & 0 & 0 & 0 \\
\hline Fíjate & 32 & $1,44 / 66,67$ & 16 & $0,86 / 33,33$ & 0 & 0 \\
\hline Hombre & 107 & $4,81 / 65,64$ & 55 & $2,95 / 33,74$ & 1 & $0,83 / 0,61$ \\
\hline Mira & 173 & $7,78 / 94,02$ & 11 & $0,59 / 5,98$ & 0 & 0 \\
\hline Mire & 16 & $0,71 / 80$ & 3 & $0,16 / 15$ & 1 & $0,83 / 5$ \\
\hline Naturalmente & 2 & $0,09 / 25$ & 4 & $0,21 / 50$ & 2 & $1,67 / 25$ \\
\hline ¿No? & 219 & $9,85 / 40,55$ & 320 & $1,72 / 59,26$ & 1 & $0,83 / 0,18$ \\
\hline Oiga & 8 & $0,36 / 72,73$ & 3 & $0,16 / 27,27$ & 0 & 0 \\
\hline Oye & 145 & $6,52 / 94,77$ & 7 & $0,37 / 4,57$ & 1 & $0,83 / 0,65$ \\
\hline Por favor & 11 & $0,49 / 44$ & 1 & $0,05 / 4$ & 13 & $10,83 / 52$ \\
\hline Por lo visto & 0 & 0 & 2 & $0,11 / 40$ & 3 & $2,5 / 60$ \\
\hline Por supuesto & 1 & $0,04 / 5,88$ & 2 & $0,11 / 11,76$ & 14 & $11,67 / 82,35$ \\
\hline Pues $^{14}$ & 424 & $19,07 / 35,07$ & 772 & $41,46 / 63,85$ & 13 & $10,83 / 1,07$ \\
\hline ¿Sabes? & 77 & $3,46 / 95,06$ & 4 & $0,21 / 4,94$ & 0 & 0 \\
\hline Sin duda & 0 & 0 & 5 & $0,27 / 25$ & 15 & $12,5 / 75$ \\
\hline Vale & 63 & $2,83 / 87,5$ & 7 & $0,37 / 9,72$ & 2 & $1,67 / 2,78$ \\
\hline Vamos & 23 & $1,03 / 23,96$ & 72 & $3,87 / 75$ & 1 & $0,83 / 1,04$ \\
\hline Verás & 3 & $0,13 / 100$ & 0 & 0 & 0 & 0 \\
\hline ¿Verdad? & 6 & $0,27 / 20$ & 24 & $1,29 / 80$ & 0 & 0 \\
\hline ¿Ves? & 17 & $0,76 / 100$ & 0 & 0 & 0 & 0 \\
\hline $\mathrm{Ya}$ & 71 & $3,19 / 87,65$ & 8 & $0,43 / 9,88$ & 2 & $1,67 / 2,47$ \\
\hline TOTAL & 2.223 & & 1.862 & & 120 & \\
\hline
\end{tabular}

Hemos sombreado aquellos marcadores cuya frecuencia pasa del 70\%. Así, por ejemplo, los marcadores conversacionales que dominan en el registro coloquial son los enfocadores de la alteridad (Martín Zorraquino \& Portolés, 1999) ‘eh?, escucha, mira/e, oye/oiga, ¿sabes?, vale, verás, ¿ves?', alguno de ellos alcanzando el dominio absoluto respecto a los tres corpus de referencia ('escucha, verás, ¿ves?'), y los marcadores metadiscursivos 'bien, ya' (Martín Zorraquino \& Portolés, 1999):

(4) E: [sí pero] no siempre $\downarrow$ no va a ser tan- es que no se trata de ser conservadora ni de na(da) $\downarrow$ se trata simplemente $\rightarrow /$ oye $\downarrow$ que cada uno viva su vida y punto/ yo soy muy demócrata $\downarrow$ mira// yo te voy a decir/ cada uno que 
viva su vida $\downarrow$ yo no tengo que arreglarle la vida a mi vecino $\uparrow$ y punto $\downarrow$ y ya está/ (L.15.A.2, Briz et al., 2002: 93)

Los marcadores ‘¿eh?, oiga’ comparten el discurso oral, pues si su frecuencia relativa en el CHCS se sumara a su frecuencia relativa en Val.Es.Co alcanzarían el 99,69\% y el 100\%, respectivamente. Coincide este resultado con las marcas de registro que proponen DPDE y Fuentes (2009) respectivamente, al considerarlos "de uso frecuente en el registro coloquial y en el discurso oral" (Briz et al., 2008, s.v. ‘¿eh?1, ¿eh 2’ y ¿eh? 3’) o “colcul. [coloquial y culto]” (Fuentes, 2009 s.v. 'oiga').

Siguiendo con el discurso oral, vemos que los marcadores metadiscursivos 'bueno, claro, pues’ y los enfocadores de la alteridad ‘¿entiendes?, fíjate, hombre, ¿no?’ dominan en los dos corpus conversacionales con suma de frecuencias relativas que superan el $98 \%$ en la mayoría de los $\operatorname{casos}^{15}$. Y además resulta interesante el caso de 'pues' que, como conversacional, presenta unas frecuencias relativa y absoluta mayores en CHCS que en Val.Es.Co. Como continuativo, Fuentes (2009, s.v. 'pues 2, pues 3') lo marca en su Diccionario con los rasgos '+coloquial +culto' y, como interactivo, con el rasgo '+ coloquial'16, por lo que nuestros resultados son los esperables:

(5) INF.— [clic] Bueno, pues estudié Medicina — la verdad es que yo tenía mis dudas entre hacer, eh, Medicina y Derecho, lo cual a mí misma también me, me sorprendía, pero luego me he dado cuenta de que le pasa a muchísima gente, ¿no? - y..., y bueno, pues, hombre, una, es una cuestión más bien puramente vocacional. (nº 7, Fernández Juncal, 2005: 137)

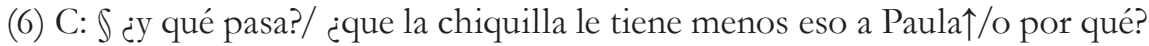

P: ¿eh?/no lo sé/porque como se ve que como se queda con ella/dirá/ si me quedo con ella $\rightarrow$ /dice/ fíjate/mamá/si es mala $\uparrow / y$ cómo nos toman el pelo los críos//dice/ ella la sube la chiquita en la silla $\uparrow / y$ le dice/no te moverás de ahí ¿eh?// (G.68.B.1 + G.69.A.1, Briz et al., 2002: 218)

Por su parte, los marcadores evidenciales (Martín Zorraquino \& Portolés, 1999) 'al parecer, en efecto, por supuesto, sin duda' dominan en el corpus escrito, llegando los dos primeros a un 100\% de frecuencias absoluta y relativa. Se corrobora así la marca '+formal' que para estos marcadores postula el DPDE (Briz et al., 2008 s.v. 'al parecer' y 'en efecto'), que destaca, además, el mayor uso de 'al parecer' en el discurso periodístico, representado en los textos de nuestro corpus escrito de referencia:

(7) Según los planes de su Patronato, el Museo del Prado va a ocupar en su ampliación el actual Museo del Ejército instalado desde hace un siglo en lo que queda del Palacio del Buen Retiro. Al parecer, los fondos del Museo del Ejército serían trasladados al Alcázar de Toledo. (El Pais, 29/11/1996, Domínguez, 2002: 563). 
Veamos ahora el registro '十formal', representado en el CHCS y en el corpus escrito: los enfocadores de la alteridad 'vamos, ¿verdad?' dominan en la conversación formal que representa el $\mathrm{CHCS}^{17}$, pero si sumamos las frecuencias relativas de los marcadores conversacionales que aparecen en los dos corpus, debemos añadir que los evidenciales 'desde luego, efectivamente, naturalmente, por lo visto' suman una frecuencia superior al 70\%. Coinciden de nuevo las consideraciones que recogen Fuentes (2009, s.v. 'desde luego') y el DPDE (Briz et al., 2008 s.v. 'efectivamente', 'naturalmente', 'por lo visto') ${ }^{18}$ :

(8) D: ese vale medio kilo seguro\

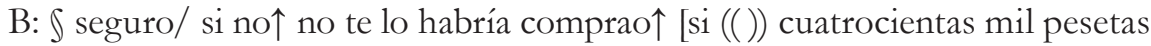
¿eh? (())]

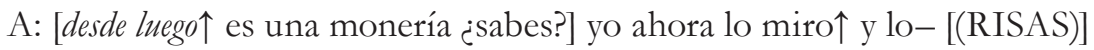

B: [(RISAS)] (RB.37.B.1, Briz et al., 2002: 230)

Cabe destacar el caso del enfocador de la alteridad 'por favor': es el único marcador de la lista de conversacionales que se sitúa en los extremos del continuum de los tipos de discurso y de registros. Frecuente en Val.Es.Co. (44\% de frecuencia relativa) y en el corpus escrito (52\%). Fuentes (2009 s.v. 'por favor') lo marca con los rasgos '+coloquial +culto +oral +escrito', consideración que concuerda con los datos de nuestro análisis.

\subsection{Estructuradores de la información}

Es predecible la mayor presencia de los estructuradores de la información (Martín Zorraquino \& Portolés, 1999) en el registro formal, ya sea en el medio oral como en el escrito. Como veremos, en la conversación coloquial suelen aparecer solo los marcadores prototípicos de esta clase - caso de 'por cierto' entre los digresores, o 'por una/otra parte' entre los ordenadores en correlación-. No obstante, encontraremos marcadores con 'cero' usos en cualquiera de los corpus de referencia de este trabajo.

\subsubsection{Ordenadores del discurso}

Varias son las características que resumirían la frecuencia en nuestros corpus de esta subclase de marcadores en el discurso: la primera incide en la escasa aparición de algunas de las unidades habituales en las listas de ordenadores ('ante todo, antes que nada', el uso del sinónimo 'término' por 'lugar' en la serie ordenadora 'en segundo/ tercer lugar/término'); la segunda se refiere a la ausencia de series correlativas de ordenadores en 'los corpus orales': estos inician una organización de su discurso con marcadores de apertura, tales como 'primero', o 'por una parte', pero después no continúan la serie (salvo algún caso aislado de 'por otra parte/lado'). La falta de planificación del discurso oral, sobre todo de la conversación coloquial, podría ser 
una de las razones achacables a esta ausencia, pues sí es significativa la aparición de marcadores de cierre (sobre todo 'al final' y 'en fin'), lo que revelaría una conciencia por parte del hablante de marcar la apertura y cierre de su discurso, pero un absoluto desconocimiento de qué, cuánto y cómo aparecerá en el interior de este.

El uso de los ordenadores del discurso en los tres corpus de referencia que hemos consultado se resume en la Tabla 3:

Tabla 3. Frecuencias absolutas y relativas (\%) de los ordenadores del discurso en su propio corpus / respecto a los tres corpus.

\begin{tabular}{|l|l|l|l|l|l|l|}
\hline Marcador & $\begin{array}{l}\text { Oral, } \\
\text { coloquial }\end{array}$ & $\mathbf{\%}$ & $\begin{array}{l}\text { Oral, } \\
\text { formal }\end{array}$ & $\mathbf{\%}$ & $\begin{array}{l}\text { Escrito, } \\
\text { formal }\end{array}$ & \% \\
\hline Al fin & 0 & 0 & 0 & 0 & 4 & $3,48 / 100$ \\
\hline Al final & 15 & $32,61 / 36,58$ & 19 & $21,35 / 46,34$ & 7 & $6,09 / 17,07$ \\
\hline Ante todo & 0 & 0 & 0 & 0 & 3 & $2,61 / 100$ \\
\hline Antes que nada & 0 & 0 & 0 & 0 & 0 & 0 \\
\hline En fin & 10 & $21,74 / 18,52$ & 29 & $32,58 / 53,70$ & 15 & $13,04 / 27,77$ \\
\hline $\begin{array}{l}\text { En primer lugar / } \\
\text { término }\end{array}$ & $1 / 0$ & $2,17 / 3,85$ & $11 / 0$ & $12,36 / 42,31$ & $14 / 0$ & $12,17 / 53,85$ \\
\hline En segundo lugar & 0 & 0 & 0 & 0 & 7 & $6,09 / 100$ \\
\hline En segundo término & 0 & 0 & 0 & 0 & 1 & $0,87 / 100$ \\
\hline $\begin{array}{l}\text { En último lugar / } \\
\text { término }\end{array}$ & $0 / 0$ & 0 & $0 / 0$ & 0 & $0 / 0$ & 0 \\
\hline Finalmente & 0 & 0 & 2 & $2,25 / 16,66$ & 10 & $8,69 / 83,33$ \\
\hline $\begin{array}{l}\text { Para empezar / } \\
\text { comenzar }\end{array}$ & $0 / 0$ & 0 & $2 / 0$ & $2,25 / 100$ & $0 / 0$ & 0 \\
\hline $\begin{array}{l}\text { Para terminar / } \\
\text { finalizar }\end{array}$ & $0 / 0$ & 0 & $2 / 0$ & $2,25 / 66,66$ & $1 / 0$ & $0,87 / 33,33$ \\
\hline Por fin & 1 & $2,17 / 14,28$ & 1 & $1,12 / 14,28$ & 5 & $4,35 / 71,43$ \\
\hline Por / de una parte & $2 / 0$ & $4,35 / 40$ & $2 / 0$ & $2,25 / 40$ & $1 / 0$ & $0,87 / 20$ \\
\hline Por un lado & 0 & 0 & 2 & $2,25 / 40$ & 3 & $2,61 / 60$ \\
\hline De un lado & 0 & 0 & 0 & 0 & 1 & $0,87 / 100$ \\
\hline Por otra parte & 2 & $4,35 / 8,69$ & 6 & $6,74 / 26,09$ & 15 & $13,04 / 65,22$ \\
\hline De otra parte & 0 & 0 & 0 & 0 & 1 & $0,87 / 100$ \\
\hline Por / de otro lado & $0 / 0$ & 0 & $3 / 0$ & $3,37 / 23,08$ & $10 / 0$ & $8,69 / 76,92$ \\
\hline Por último & 0 & 0 & 1 & $1,12 / 16,66$ & 5 & $4,35 / 83,33$ \\
\hline Por su parte & 0 & 0 & 0 & 0 & 1 & $0,87 / 100$ \\
\hline Primero & 14 & $30,43 / 45,16$ & 9 & $10,11 / 29,03$ & 8 & $6,96 / 25,81$ \\
\hline Segundo & 1 & $2,17 / 33,33$ & 0 & 0 & 2 & $1,74 / 66,66$ \\
\hline Tercero & 0 & 0 & 0 & 0 & 1 & $0,87 / 100$ \\
\hline TOTAL & 46 & & 89 & & 115 & \\
\hline & & & & & & \\
\hline
\end{tabular}

Los ordenadores del discurso que pasan del 70\% se hallan mayoritariamente en el corpus escrito: son los casos de 'al final, ante todo, en segundo lugar/término, finalmente, por fin, de un lado, de otra parte, por otro lado, por último, por su parte, tercero'. Muchos de ellos no aparecen en ninguno de los corpus orales consultados. El único ordenador que aparece exclusivamente en CHCS es 'para empezar', marcado como frecuente en el discurso oral por el DPDE (Briz et al., 2008 s.v. 'para empezar'): 
(9) INF.- Sí. Para empezar, yo, cuando estudiaba, había dos mil alumnos [carraspeo] (n¹1, Fernández Juncal, 2005: 216)

Y en Val.Es.Co son los ordenadores de apertura, 'primero', y de cierre, 'al final', los únicos que superan el 30\% de frecuencias absoluta y relativa. De hecho, 'primero' supera en Val.Es.Co. a las frecuencias del registro '+formal' oral y escrito, corroborando así la marca de registro que de este ordenador hace el DPDE (Briz et al., 2008 s.v. 'primero... segundo... tercero'):

(10) B: ahí ten- ahí tenemos el ordenador y el internet/ oye/ por internet/ se recibe/ bueno/ primero ya lo hemos- ya lo hemos comentado/ por la televisión $\uparrow /$ se anuncian todos estos [brujos entre comillas=] (XP.48.A.1, Briz et al., 2002: 358)

Y 'al final' aparece más frecuentemente en los corpus orales que en el escrito, con una mayor frecuencia relativa en el $\mathrm{CHCS}^{19}$ :

(11) A: ¿¿QUÉ estuvisteis/una mañana con ellos? $\int$

$\mathrm{V}: \int$ sí sí/ comimoos/ y estuvimos unas- unas horas/ solo/ solo unas horas $\int$ A: $₫ y$ al final/ bien/ ¿no? $\int$

$\mathrm{V}: \int$ sí $\downarrow$ muy bien/ me reí mucho/ hablamos/ o sea estuvi- estuvimos muy a gusto/// y hemos quedado que- ya veremos ¿no?/ (IH.340.A.1, Briz et al., 2002: 381)

(12) inf.- Porque es lo más seguro. Y realmente el trabajo, más que por vocación, te tiene que servir para ganar dinero y para tener una estabilidad. Entonces no hay que confundir una cosa con la otra. Entonces yo de ocho a tres gano dinero para vivir, y luego, a las tres de la tarde, hago lo que me da la gana. Y de la otra forma estaría trabajando catorce horas diarias en algo que me gusta pero que, al final, dentro de diez años, ya ni te gusta (n³, Fernández Juncal, 2005: 64)

En los corpus de registro formal aparecen más ordenadores de apertura y estructuras 'en serie discursiva' (Garcés, 2008), aunque siempre son más numerosos en el corpus escrito. Las correlaciones solo tienen el marcador de apertura en el CHCS, que prefiere continuarse con marcadores temporales como 'después' o 'a partir de ahí';

(13) inf.- O sea, lo que se ve es, primero que el espíritu universitario no es el mismo, y, a partir de ahí, la vida universitaria no es la misma, (nº), Fernández Juncal, 2005: 178-179)

Sí hay correlación, en cambio, en el discurso escrito: 
(14) Hay al menos tres motivos para una aseveración así. Primero, la doctrina al fondo es que el consumo y el empleo son equivalentes lógicos, que uno conlleva al otro. [...] Segundo, y gracias al triunfo innegable de la filosofía del consumo, el valor del dinero hoy se estima en gran medida según su poder de adquirir lo que hay que comprar por deferencia a los anuncios que oímos todo el día y en todo sitio. [...]

$\mathrm{Y}$, tercero $^{20}$, por supuesto, hay que tomar en cuenta que el consumo está destruyendo el planeta en que vivimos. (El País, 22/1/1997, Domínguez, 2002: 591)

El carácter anafórico de los ordenadores, incluido el del primer miembro de la correlación, parece claro en este ejemplo, si tenemos en cuenta que responden al anuncio de enumeración hecho en el enunciado precedente ('al menos tres motivos'). Igualmente clara queda su función ordenadora, doblemente especificada en casos como el de este ejemplo (14), donde el último párrafo no solo se introduce con el ordenador 'tercero' sino que, además, aparece el conector ' $y$ ' que ha añadido a su valor argumentativo prototípico de adición el valor discursivo de cierre (Garcés, 1997, 2001).

Nos centramos ahora en el uso de los ordenadores de cierre. Como se puede observar en la Tabla 3, su frecuencia es mucho mayor en el corpus escrito, conclusión esperable dada la característica de su mayor planificación. Algunos, como 'al fin', de aparición exclusiva:

(15) "Busque por acá en qué se le haga merced": esta fue, al fin, la lacónica respuesta que el imperio, por el cual a punto estuvo el joven Cervantes (el auténtico Quijote) de ofrendar su propia vida, propinó a su deseo y a su derecho de una existencia nueva en un nuevo mundo.

Otros, sin embargo, comparten el registro '+formal' y aparecen en CHCS: son los casos de 'finalmente, para terminar y por último':

(16) ENC.- Y para terminar, ¿me puedes decir el nombre de una persona famosa que te parezca un modelo lingüístico, es decir, una persona que, cuando la oyes hablar dices: “QQué bien habla!"? (n¹, Fernández Juncal, 2005: 41)

Y el marcador 'en fin' aparece también con una frecuencia absoluta alta en el corpus coloquial:

$(17) \mathrm{D}: \mathbb{\int}(())$ es que/ no sé $\downarrow$ me sabe mal/desprenderme $\uparrow$ pero yo quisiera $\rightarrow$ que me lo tasara $\uparrow$ porque $\rightarrow \llbracket$

C: $₫$ claro $\$$ 
B: $\int$ ves y que [te lo tasen por el centro]

D: [porque es quee] estamos un poco apuraos $\uparrow$ pero en fin $\downarrow$ ee yo es que- por lo menos $\uparrow$ usted tasémelo a ver quee (( ))S (RB.37.B.1., Briz et al., 2002: 228 229)

González Fernández (1996) señala el valor prototípico de 'cierre discursivo’ que tiene el marcador 'por último' que, como en el ejemplo siguiente, "simplemente ubica el elemento que, en una serie discursiva, ocupa el último lugar”:

(18) Tras leer la reproducción del artículo Polanco publicado en el periódico El Mundo y firmado por don Pedro J. Ramírez - un nuevo y claro exponente del periodismo de investigación, riguroso, imparcial, objetivo y desapasionado-, deseo exponerle algunas medidas para paliar el desolador panorama que el señor Ramírez nos dibuja:

1. $[\ldots]$

2. $[\ldots]$

8. Y,por último, prohibir todos aquellos medios de comunicación que no utilicen la falacia, la demagogia, el descalificativo y el confusionismo como medio de expresión para que los ciudadanos no tengan elementos de comparación y puedan, de esta manera, distinguir el verdadero periodismo del panfleto insidioso. (El País, 27/1/1997, Domínguez, 2002: 592-593)

El resto de marcadores de cierre añaden a su función ordenadora un valor conclusivo, de recapitulación (Garcés, 1997) y además, dependiendo del contexto en el que se hallen, una serie de valores modales, como la 'resignación' ${ }^{21}$-ejemplos (15), 'al fin', y (17), 'en fin'- o el 'cansancio' - ejemplos (11) y (12), 'al final'- del hablante. Quizás sea esta polivalencia la que explique su mayor frecuencia en todos los discursos y registros.

\subsubsection{Digresores}

En este subgrupo solo el marcador 'por cierto' es el más frecuente en todos los corpus, sin estar marcado, por tanto, por ningún registro (Portolés, 1998; Martín Zorraquino \& Portolés, 1999). El resto de digresores no tiene presencia alguna en los corpus orales y solo una presencia residual en el corpus escrito (Briz et al., 2008 s.v. 'por cierto, a propósito, dicho sea de paso, entre paréntesis'): 
Tabla 4. Frecuencias absolutas y relativas (\%) de los digresores en su propio corpus / respecto a los tres corpus.

\begin{tabular}{|l|l|l|l|l|l|l|}
\hline Marcador & $\begin{array}{l}\text { Oral, } \\
\text { coloquial }\end{array}$ & $\mathbf{\%}$ & $\begin{array}{l}\text { Oral, } \\
\text { formal }\end{array}$ & $\mathbf{\%}$ & $\begin{array}{l}\text { Escrito, } \\
\text { formal }\end{array}$ & \% \\
\hline A propósito & 0 & 0 & 0 & 0 & 1 & $9,09 / 100$ \\
\hline A todo esto & 0 & 0 & 0 & 0 & 0 & 0 \\
\hline Dicho sea de paso & 0 & 0 & 0 & 0 & 1 & $9,09 / 100$ \\
\hline Entre paréntesis & 0 & 0 & 0 & 0 & 0 & 0 \\
\hline Por cierto & 3 & $100 / 12$ & 13 & $100 / 52$ & 9 & $81,82 / 36$ \\
\hline TOTAL & $\mathbf{3}$ & & 13 & & 11 & \\
\hline
\end{tabular}

(19) S: porquee- porque pasa de todo// no $\downarrow$ porque no haya podido no/si creo qu'él me pagó y todo/ laa- la plaza

$\mathrm{J}:[(($ este año se ha pagao todo)) $]$

C:[por cierto/ ahora que dices] eso/ vamos a hacer un listado de los que han pagao $\uparrow$

S: $\mathrm{mm}$ mm (AP.80.A.1, Briz et al., 2002: 156)

(20) INF. - El campus ni se soñaba. El campus... lo concibe, lo gesta L'. estamos enfrente de su calle por cierto- (n¹1, Fernández Juncal, 2005: 217)

(21) Por ello, la prensa, la televisión y la radio nos martirizan, a los que apenas nos sobran unas horas para dormir, con temas tan triviales como el que aparezcan en carteles publicitarios los pechos de una dama. Que si el queso de tetilla que si el bebé feliz entre unos pechos protectores.

Por cierto, me parecen unos pechos estupendos y tan maternales que en mí lo único que suscitan es ternura (El País 2/3/1007, Domínguez, 2002: 639)

\subsubsection{Comentadores}

Finalmente, en el grupo de los comentadores (Martín Zorraquino \& Portolés, 1999) hemos de señalar que 'de momento' es el marcador preferido por los corpus orales $-76,47 \%$ suman sus frecuencias relativas de aparición-, aunque también aparece en el corpus escrito. El resto de comentadores tiene un dominio absoluto en el corpus escrito. Solo el comentador 'hasta aquí' parece exclusivo del registro + coloquial, pero hemos localizado una sola muestra en Val.Es.Co. y, por tanto, no podemos sacar conclusiones determinantes sobre él: 
Tabla 5. Frecuencias absolutas y relativas (\%) de los comentadores en su propio corpus / respecto a los tres corpus.

\begin{tabular}{|l|l|l|l|l|l|l|}
\hline Marcador & $\begin{array}{l}\text { Oral, } \\
\text { coloquial }\end{array}$ & $\mathbf{\%}$ & $\begin{array}{l}\text { Oral, } \\
\text { formal }\end{array}$ & $\mathbf{\%}$ & $\begin{array}{l}\text { Escrito, } \\
\text { formal }\end{array}$ & $\mathbf{\%}$ \\
\hline Así las cosas & 0 & 0 & 0 & 0 & 0 & 0 \\
\hline Dicho esto/eso & $0 / 0$ & 0 & $0 / 0$ & 0 & $1 / 0$ & $3,57 / 100$ \\
\hline Hasta ahí & 1 & $11,11 / 100$ & 0 & 0 & 0 & 0 \\
\hline Hasta aquí & 0 & 0 & 0 & 0 & 1 & $3,57 / 100$ \\
\hline Hasta ahora & 0 & 0 & 0 & 0 & 1 & $3,57 / 100$ \\
\hline Por el momento & 0 & 0 & 0 & 0 & 2 & $7,14 / 100$ \\
\hline De momento & 7 & $77,77 / 41,18$ & 6 & $100 / 35,29$ & 4 & $14,28 / 23,53$ \\
\hline Pues bien & 1 & $11,11 / 5$ & 0 & 0 & 19 & $67,86 / 95$ \\
\hline TOTAL & $\mathbf{9}$ & & 6 & & 28 & \\
\hline
\end{tabular}

22) V: [te digo yo] que como se plantee/ según como se plantee

A: según como se plantee la ley de los presupuestos

$\mathrm{V}$ : ¿eeh? o sea que/ de moMENto $\uparrow /$ eso/ la idea es esa/ qu'el alcalde sea mayoritario// [para evitar ((eso))// oye ¿este coñac?] (J.82.A.1, Briz et al., 2002:185)

\section{CONCLUSIONES}

Los resultados que hemos obtenido en el apartado anterior nos permiten esbozar unas primeras conclusiones sobre el uso de los marcadores del discurso en la variedad geográfica del español de España. Partimos de la asunción de que, para que estas conclusiones resulten determinantes debemos ampliar la muestra a otros corpus de referencia, propósito para futuros trabajos, tal como ya hemos indicado. En resumen, a) una conclusión predecible que de hecho se da es la tendencia de los estructuradores de la información a aparecer en los discursos escritos, en tanto que los conversacionales tienen su sede en los discursos orales. Así lo hemos comprobado en los tres corpus de referencia. Sin embargo, mediante la introducción de la variable de registro, hallamos que los conversacionales evidenciales dominan en el registro formal, mayoritariamente en el discurso escrito -'al parecer, en efecto'- aunque también presentes en el discurso oral formal -'desde luego, efectivamente, naturalmente'-; por su parte, los ordenadores de cierre 'en fin' y 'al final' y el apertor 'primero' aparecen con una cierta frecuencia en el registro oral coloquial; y b) cuando se realiza un análisis de frecuencias como el que hemos llevado a cabo en este trabajo, observamos que son pocos los marcadores de las listas habituales que tienen un uso verdaderamente significativo. Así lo vemos en el caso de los digresores, que concentran su uso en el prototípico 'por cierto'; o de los comentadores, para los que los tres corpus analizados prefieren 'de momento'.

Conclusiones como estas nos permiten esbozar una propuesta de una nueva lista de marcadores que tenga en cuenta las variables de tipo de discurso y de registro: 
Tabla 6. Nueva lista de marcadores propuesta, en la que se desglosan tipos de discurso y registros.

\begin{tabular}{|c|c|c|c|}
\hline Marcador & Oral, coloquial & Oral, formal & Escrito, formal \\
\hline Conversacional & $\begin{array}{l}\text { bien } \\
\text { bueno }^{22} \\
\text { claro } \\
\text { desde luego } \\
\text { ¿eh? } \\
\text { ¿entiendes? } \\
\text { fijate } \\
\text { hombre } \\
\text { mira/mire } \\
\text { ¿no? } \\
\text { oye/oiga } \\
\text { por favor } \\
\text { pues } \\
\text { ¿sabes? } \\
\text { vale } \\
\text { ¿ves? } \\
\text { ya }\end{array}$ & $\begin{array}{l}\text { bueno } \\
\text { ¿entiendes? } \\
\text { ¿no? } \\
\text { por lo visto } \\
\text { pues } \\
\text { vamos } \\
\text { ¿verdad? }\end{array}$ & $\begin{array}{l}\text { al parecer } \\
\text { efectivamente } \\
\text { en efecto } \\
\text { por lo visto } \\
\text { por supuesto } \\
\text { sin duda }\end{array}$ \\
\hline Ordenador & $\begin{array}{l}\text { al final } \\
\text { por una parte } \\
\text { primero }\end{array}$ & $\begin{array}{l}\text { al final } \\
\text { en fin } \\
\text { en primer lugar } \\
\text { para empezar } \\
\text { para terminar } \\
\text { por una parte } \\
\text { por un lado }\end{array}$ & $\begin{array}{l}\text { al fin } \\
\text { en primer lugar... en segundo } \\
\text { lugar } \\
\text { finalmente } \\
\text { por fin } \\
\text { por otra parte } \\
\text { por un lado... por otro lado } \\
\text { por último }\end{array}$ \\
\hline Digresor & por cierto & por cierto & por cierto \\
\hline Comentador & de momento & de momento & $\begin{array}{l}\text { de momento } \\
\text { pues bien }\end{array}$ \\
\hline
\end{tabular}

Fieles a la aplicación pretendida en esta propuesta, anunciada desde el inicio del artículo, si tenemos en cuenta las frecuencias de aparición de los marcadores en los tipos de discurso oral y escrito y en los registros coloquial y formal, podremos establecer nuevas listas, como la anterior, para la enseñanza-aprendizaje de los marcadores en la clase de español como segunda lengua o como lengua extranjera. Tomemos como ejemplo el nivel umbral B1 y los marcadores que incluye el ya dicho PCIC (Instituto Cervantes, 2006 y en línea) para aprender en este nivel:

Tabla 7. Propuesta del PCIC para la enseñanza de marcadores discursivos en el nivel B1.

\begin{tabular}{|l|l|l|l|l|}
\hline Marcador & Conversacional & Ordenador & Digresor & Comentador \\
\hline Nivel B1 & claro & en primer lugar & & pues \\
\hline & ¿'entiendes? & en segundo / tercer... lugar & & \\
\hline & $¿$ ¿sabes? & en conclusión & & \\
\hline & ¿ves? & finalmente & & \\
\hline & & para terminar & & \\
\hline & & por otro lado & & \\
\hline & & por otra parte & & \\
\hline & por un lado & & \\
\hline & & por una parte & & \\
\hline
\end{tabular}


Observamos que se centra particularmente en los estructuradores de la información, aunque no incluye digresores - estos aparecen en el PCIC a partir del nivel B2- y solo un comentador, 'pues' ${ }^{23}$. En cuanto a los marcadores conversacionales, solo incluye unos pocos marcadores de control del contacto.

A partir de nuestro estudio de marcadores frecuentes, podríamos aportar a la lista del PCIC datos sobre el tipo de discurso - oral o escrito- y de registro -coloquial, formal- en los que son más susceptibles de aparecer, así como ampliar un poco más la relación. 


\section{REFERENCIAS BIBLIOGRÁFICAS}

Albelda, M. (2005). El tratamiento de las partículas discursivas en algunas gramáticas y manuales del español para extranjeros. Actas del XV Congreso Internacional de ASELE. Sevilla: Universidad de Sevilla, 111-119.

Blakemore, D. (1987). Semantic constraints on relevance. Oxford: Blackwell.

Briz, A. (1993a). Los conectores pragmáticos en español coloquial (I): Su papel argumentativo. Contextos, XI/21-22, 145-188.

Briz, A. (1993b). Los conectores pragmáticos en la conversación coloquial (II): Su papel metadiscursivo. Español Actual, 39-56.

Briz, A. (1998). El español coloquial en la conversación. Esbozo de pragmagramática. Barcelona: Ariel.

Briz, A. \& Grupo Val.Es.Co. (2002). Corpus de conversaciones coloquiales. Madrid: Arco Libros.

Briz, A., Pons, S. \& Portolés, J. (2008). Diccionario de partículas discursivas del español [en línea]. Disponible en: www.dpde.es

Domínguez, M. N. (2002). La organización del discurso argumentativo: Los conectores. Salamanca: Ediciones Universidad.

Domínguez, M. N. (2010). Los marcadores del discurso y los tipos textuales. En Ó. Loureda \& E. Acín (Coords.), Los estudios sobre marcadores del discurso en español, boy (pp. 359-413). Madrid: Arco Libros.

Domínguez, M. N. (2011). Comentadores del discurso. En R. González Ruiz \& C. Llamas Saíz (Eds.), Gramática y discurso. Nuevas aportaciones sobre partículas discursivas del español (pp. 39-56). Pamplona: EUNSA.

Eggins, S. (2002). Introducción a la Lingüistica Sistémica. La Rioja: Universidad de La Rioja.

Fernández Juncal, C. (2005). Corpus de Habla Culta de Salamanca (CHCS). Burgos: Instituto Castellano y Leonés de la Lengua.

Fuentes. C. (1987). Enlaces extraoracionales. Sevilla. Alfar.

Fuentes, C. (2009). Diccionario de conectores y operadores del español. Madrid: Arco Libros.

Garcés, M. P. (1997). Procedimientos de ordenación en los textos escritos. Romanistisches Jabrbuch, 48, 296-315. 
Garcés, M. P. (2001). Organización informativa en el discurso y conexión. A propósito de los marcadores discursivos 'por otra parte / por otro lado'; 'de otra parte / de otro lado'. Verba, 28, 281-303.

Garcés, M. P. (2008). La organización del discurso: Marcadores de ordenación y de reformulación. Madrid/Frankfurt: Iberoamericana/Vervuert.

González Fernández, M. J. (1996). Conectores discursivos, con especial referencia al español de México (orden, expectativas y valoración). Tesis Doctoral, Universidad de Salamanca, Salamanca, España.

Holgado, A. (2014). Pragmática lingüística aplicada al español para extranjeros, con especial atención a los marcadores discursivos: El Diccionario de marcadores discursivos para estudiantes de español como lengua extranjera. Tesis Doctoral, Universidad de Salamanca, Salamanca, España.

Instituto Cervantes (2006). Plan Curricular del Instituto Cervantes [en línea]. Disponible en: http://www.cervantes.es/lengua_y_ensenanza/aprender_espanol/plan_ curricular_instituto_cervantes.htm

Marimón Llorca, C. (2008). Análisis de textos en español. Teoría y práctica. Alicante: Publicaciones de la Universidad de Alicante.

Martín Zorraquino, M. A. \& Portolés, J. (1999). Los marcadores del discurso. En I. Bosque \& V. Demonte (Dirs.), Gramática descriptiva de la lengua española (pp.4051-4213). Madrid: Espasa.

Mederos, H. (1988). Procedimientos de cohesión en el español actual. Santa Cruz de Tenerife: Cabildo insular de Tenerife.

Montolío, E. (2003). Es una buena periodista, en principio. Sobre el operador discursivo en principio y su función modalizadora en el discurso periodístico. Español Actual, 79, 45-58.

Pons, S. (1998). Conexión y conectores. Estudio de su relación en el registro informal de la lengua. Anexo no XXVII de la revista Cuadernos de Filología. Valencia: Universitat de València.

Portolés, J. (1998). Marcadores del discurso. Barcelona: Ariel.

Portolés, J. (2004). Pragmática para hispanistas. Madrid: Síntesis.

Taranilla, R. (2008). A lo que iba: Evolución y uso de un marcador de regresión. En I. Olza, M. Casado \& R. González (Eds.), Actas del XXXVII Simposio Internacional de la Sociedad Española de Lingüistica (SEL) (pp.825-836). Pamplona: Servicio de Publicaciones de la Universidad de Navarra.

Tusón, A. (1997). Análisis de la conversación. Barcelona: Ariel. 


\section{NOTAS}

1 Sobre las convenciones de transcripción de las conversaciones coloquiales usadas en los ejemplos, véase Briz y Val.Es.Co. (2002: 28-36).

$2 \mathrm{Al}$ respecto, véase Briz (1993a, 1993b).

3 Usamos el término de Taranilla (2008) en su estudio del marcador 'a lo que iba'.

4 En su estudio de 'en principio', Montolío (2003: 46) señala que "este marcador está ampliamente extendido en todos los registros del español".

5 Problema que se acrecienta si se incluyeran todos los marcadores usados en el ámbito hispánico por hablantes nativos de español: como ejemplo mínimo, tomemos el caso de 'vale', como marcador de acuerdo en el español de España, que se correspondería con 'dale' en el español de Argentina y Chile, 'órale' en el español de México, 'okei', 'oka' en el español del Caribe... Y no en todas las zonas de esos países se usan estos marcadores.

6 Briz (1998) resume la bibliografía sobre esta distinción en 20 (Nota 2).

7 Intentar una cierta exhaustividad bibliográfica es todo un atrevimiento, pues implica siempre algún olvido. No obstante, remitimos a Domínguez (2010) para una revisión bibliográfica más o menos extensa de los estudios sobre marcadores del discurso elaborados hasta la primera década del presente siglo.

8 En esta muestra aleatoria que hemos tomado como ejemplo, observamos que el PCIC concluye el aprendizaje de los marcadores de control de contacto en el nivel B1. Si existe una correlación entre la frecuencia de uso de estos marcadores y su aprendizaje, el resultado esperable en nuestro estudio será una frecuencia alta en el uso de estos marcadores, como de hecho se ha dado.

9 Según las exigencias editoriales del diario ElPaís durante el período de recogida de datos: noviembre de 1996-abril de 1997.

10 Sobre el tipo de conversaciones, la metodología de la muestra y los criterios de transcripción, remitimos a la Introducción de Briz et. al. (2002: 1-40).

11 No incluimos los conectores, presentes en cualquier secuencia argumentativa de cualquier tipo de discurso, oral o escrito, coloquial o formal.

12 Ampliaremos la lista de comentadores con la que elaboramos en Domínguez (2011).

13 Dada la polivalencia de algunos marcadores, como los casos de 'bueno' y 'bien', hemos unificado sus funciones conversacionales como marcadores de modalidad deóntica y marcadores metadiscursivos.

14 Como en el caso anterior de los marcadores polivalentes 'bueno' y 'bien', para el caso de 'pues' hemos unificado los casos en los que funciona como marcador conversacional metadiscursivo de control del mensaje (Briz, 1998) o continuativo (Fuentes, 2009 s.v. 'pues 2') y como ordenador discursivo interactivo (Fuentes, 2009 s.v. 'pues 3'), y hemos excluido los casos en los que funciona como conector, con valores argumentativos de consecuencia o de oposición.

15 De estos marcadores, 'claro' solo llega al 73,54\% de suma de frecuencias relativas en los dos corpus conversacionales, pero ya es una cifra alta de frecuencia.

16 En la recogida de datos para este estudio, hemos unificado ambas funciones. Al respecto, véase nota 13 .

$17 \mathrm{Al}$ respecto de estos marcadores, el DPDE los considera orales, 'vamos' con mayor tendencia al registro coloquial y ‘ंverdad?' sin tendencia a un registro concreto (Briz et al., 2008, s.v. 'vamos, ¿verdad? 1, ¿ंverdad? 2').

18 De 'naturalmente', además, indica este Diccionario su mayor presencia en textos escritos, circunstancia que no se da en nuestro análisis, ya que es en el CHCS donde aparece con el doble de frecuencia que en Val.Es.Co. y en el corpus escrito.

19 Si sumamos las frecuencias relativas de 'al final' en el CHCS y en el corpus escrito tenemos que el resultado dobla a la de Val.Es.Co. Ya el DPDE indicaba la mayor presencia de este ordenador en el registro formal de la lengua (Briz et al., 2008 s.v. 'al final'), sin embargo no debemos desdeñar su frecuencia absoluta en el corpus coloquial consultado, ya que es la más alta de los ordenadores usados en él. 
20 Véase en este ejemplo el único caso de correlación discursiva de tres miembros que hemos encontrado en los corpus analizados: 'primero... segundo....y tercero'.

21 González Fernández (1996: 361) utiliza el concepto pragmático de "valor cancelatorio de resignación” para explicar el funcionamiento del marcador 'en fin' en algunos casos como el del ejemplo (17), en el que se produce la "cancelación de las expectativas del hablante, que acepta el curso de los acontecimientos".

22 Algunos marcadores se repetirán en las diferentes columnas -'bueno, ¿entiendes?, ¿no?, pues, por lo visto'-, lo que demostraría su indiferencia ante el registro -'bueno, ¿entiendes?, ¿no?, pues', presentes en el discurso oral coloquial y formal- o ante el tipo de discurso -'por lo visto', presente en el registro formal de la lengua, ya sea oral o escrita-.

23 El ejemplo que ofrece el PCIC del 'pues' comentador coincide con los casos de 'pues' que en nuestro trabajo hemos incluido en el apartado de marcadores conversacionales. 